\title{
Aktuelle Entwicklungslinien in der schwedischen Quartärgeologie ${ }^{1}$ )
}

\author{
Von TAge Nilsson, Lund
}

A bstract. The present paper is a translation of a lecture held at the 7 th meeting of Nordic geologists in Stockholm on July 4, 1958. It treats in a very brief outline some actual trends of development as regards Quaternary geology in Sweden. Special stress is laid on the progress made in the last decade. The material has been divided into the following sections: geological mapping, study of interglacial deposits, glacial geology, varve chronology, study of the changes of level, history of vegetation and climate, radiocarbon datings. Certain new C 14 determinations are given.

\section{In ha $1 \mathrm{t}$}

1. Kartierungsarbeiten der Schwedischen Geologischen Landesanstalt

2. Interglaziale Ablagerungen

3. Glazialgeologie

4. Warvenchronologie

5. Niveauveränderungen

6. Vegetations- und Klimageschichte

7. Altersbestimmungen mit der Radiokarbonmethode

Während des letzten Jahrzehnts verlor die schwedische Quartärgeologie einen ihrer größten Männer: Lennart von Pos'. Er starb Neujahr 1951, nachdem bereits 8 Jahre früher ein anderer führender Quartärgeologe, Gerard DE GEER, von uns gegangen war. Die Initiative, die diese beiden ergriffen haben und die Anregungen, die von ihnen ausgegangen sind, geben indessen noch immer der schwedischen Quartärgeologie ihr Gepräge. Warvenchronologie, Glazialgeologie, die Erforschung der Niveauveränderungen, Datierung mit Hilfe der Pollenanalyse, das Studium der Vegetationsgeschichte und der Klimaentwicklung während der spätquartären Zeit, alles das wird noch heute in großem Ausmaße nach den Richtlinien betrieben, die von den genannten Forschern bestimmt wurden. Nach und nach sind jedoch mehrere neue Züge ins Bild hineingekommen. Im Augenblick möchte ich nur die Altersbestimmung mit der Radiokarbonmethode nennen.

\section{Kartierungsarbeiten der Schwedischen Geologischen Landesanstalt}

Die Kartierungsarbeiten der Schwedischen Geologischen Landesanstalt (SGU) waren und sind noch heute u. a. für die schwedische Quartärgeologie von grundlegender Bedeutung. Die Detailkartierung und die Herausgabe von Kartenblättern im Maßstab $1: 50000$ (kombinierte Untergrund- und Bodenkarten, Ser. Aa) sind bis nach Mittelschweden vorgedrungen. Vereinzelte Karten in gleichem Maßstab von südlicheren Teilen des Landes sind ebenfalls im Laufe der letzten Jahre herausgegeben worden. Teilweise handelt es sich um neue Auflagen der älteren Kartenblätter, die in gleichem oder kleinerem Maßstab erschienen waren. Die Herausgabe einer neuen Serie von noch ausführlicheren, sog. agrogeologischen Karten (Ser. Ad) wurde in Schonen begonnen, und zwar mit 9 Blättern im Maßstab 1:20000, ausgearbeitet von Gunnar Екsтвӧм. In Nordschweden kann eine Kartierung nicht mit gleicher Ausführlichkeit wie in Mittel- und Südschweden durchgeführt werden, wenn die Karten in absehbarer Zeit zur Verfügung stehen sollen. Hier ist

1) Vortrag, gehalten anläßlich der 7. nordischen Geologentagung in Stockholm am 4. 7. 1958. Behandelt wurden nur die wichtigsten Fortschritte des letzten Jahrzehnts. Die Übertragung ins Deutsche wurde von Frau Hannelore Håkansson, Lund, ausgeführt. 
man dazu übergegangen, geologische Übersichtskarten in relativ kleinem Maßstab, sog. „Länskarten“2), herauszugeben. Diese werden in Ser. Ca publiziert. Quartärgeologische Karten dieser Art erschienen während des letzten Jahrzehnts für Kopparbergs län (1951, Gösta LundQvist; Maßstab $1: 250$ 000) und Värmlands län (1958, Jan LundQvist; Maßstab $1: 200000)$. Im Erscheinen begriffen ist eine quartärgeologische Karte von Norrbotten (Erik Fromm).

Bezeichnend für die geologische Kartierung der letzten Jahre ist die Arbeit, diè nunmehr auf die genaue Charakterisierung und Aufteilung der Bodenarten verwandt wird. Dieses gilt in erster Linie für Eкsтröм's sehr ausführliche, agrogeologische Kartenblätter, die besonders dazu bestimmt sind, der Landwirtschaft zu dienen. Es gilt aber auch für sonstige geologische Karten. Vor allem ist die Systematisierung der Moränenbodenarten Gegenstand großen Interesses geworden (Gösta LundQvist). Moränenböden spielen eine besonders wichtige Rolle in unserem Lande, und eine Aufteilung der hierhergehörenden Bodenarten ist von großer Bedeutung für die Forstwirtschaft.

Eine übersichtliche Karte über die quartären Ablagerungen von ganz Schweden wurde $\mathrm{zu}$ dieser Tagung von Gösta Lundevist ausgearbeitet (SGU, Ser. Ba; Maßstab 1: $\left.1000000)^{3}\right)$.

\section{Interglaziale Ablagerungen}

Das Vorkommen von evtl. interglazialen Ablagerungen in Schweden ist seit langem lebhaft diskutiert worden. Es scheint, als habe die Forschung des letzten Jahrzehnts einen endgültigen und positiven Bescheid zu dieser Frage gegeben, wenigstens für die Mehrzahl der nordschwedischen Funde. Zahlreiche Vorkommen mit submoränalen Bildungen in Nordschweden müssen in Übereinstimmung damit als interglazialen Alters betrachtet werden. Man ist auf zwei oder drei völlig verschiedenen Wegen zu diesem Resultat gekommen. Bereits 1948 konnte Lindroth nachweisen, daß die fossilen Insekten der fraglichen interglazialen Ablagerungen zum größten Teil aus Arten bestanden, deren heutige Verbreitung es unwahrscheinlich erscheinen läßt, daß sie postglazial sind. Während der allerletzten Jahre sind eine Reihe von Altersbestimmungen mit der $C^{14}$-Methode ausgeführt worden, die zum gleichen Resultat führten (Gösta LundQvist 1955, 1957; Jan LuNDQvist 1955, 1958; ỚstLund 1957). Sie ergaben folgende Werte:

O j e (Dalarna), submoränaler Fichtenstamm: über 40000 Jahre (vor heute)

B oliden (Västerbotten), submoränale Pflanzenreste: über 24000 Jahre

Bollnäs (Hälsingland), submoränale Gyttja: über 30000 Jahre

Pilgrimstad (Jämtland), Fundort interglazialer Mammutreste in primärer Lagerung; submoränale Pflanzenreste: über 39000 Jahre

V å 1 b a ck e n (Jämtland), submoränale Pflanzenreste: über 37000 Jahre

F r y k s t a (Värmland), Fichtenholz aus einer spätquartären glazifluvialen Ablagerung: über 39000 Jahre (offenbar umgelagert).

Sämtliche Vorkommen haben ein Alter, das außerhalb des Bereichs der Messungs-Methode liegt.

An einigen Stellen (Långsele, Bollnäs, Oje) wurden außerdem pollenanalytische Untersuchungen durchgeführt. Bei Óje konnte Erdtman (Lundovist 1955) u. a. Pollenkörner der Lärche (Larix) identifizieren. Dieser Baum gehört ja nicht der spontanen postglazialen Flora Schwedens an.

2) Schwed. län $=$ Regierungsbezirk.

3) In dem im Erscheinen begriffenen "Atlas über Schweden“ sind außerdem mehrere Karten enthalten, die verschiedene quartärgeologische Erscheinungen darstellen. 
Die Funde von $\mathrm{M}$ a $\mathrm{m} \mathrm{m}$ u $\mathrm{t} \mathrm{r}$ e s t e $\mathrm{n}$ haben allmählich recht beträchtlich zugenommen. Sie stammen zum größten Teil aus Nordschweden und wurden in der Regel in Grundmoräne, in sekundärer Lagerung, angetroffen. Das Mammut dürfte in interglazialer Zeit in diesen nördlichen Gegenden gelebt haben.

\section{Glazialgeologie}

Die Glazialgeologie nimmt aus natürlichen Gründen einen bedeutenden Platz in der schwedischen Quartärgeologie ein. Hier ist zuerst die von Erik LJUNGNER und seiner Schule (Gunnar Johnsson, Martin Markgren, Åke Mattsson) ausgearbeitete und angewandte Eis schrammenmethodik (schwed. „hällanaly s“) ${ }^{4}$ ) zu nennen. Diese Methode geht darauf aus, hinsichtlich der Beschaffenheit und der Bewegungsänderungen des Eises sowie der Chronologie und Dauerhaftigkeit der Eisbewegung Schlußfolgerungen zu ziehen. Dies geschieht mit Hilfe der Eisschrammen und anderen Spuren der Eisbewegung auf dem felsigen Untergrund, deren Richtungsbestimmung und qualitativer sowie quantitativer Abschätzung. Durch Verwendung seiner Eisschrammenmethodik in den schwedischen Fjällgebieten glaubte LJungnen u. a. feststellen zu können, daß die letzte Vereisung in zwei Stadien zerfiel: eine ältere sog. Primvereisung und eine jüngere Hauptvereisung, in welch' letzterer das Eis eine wesentlich größere Ausbreitung hatte als in dem ersten Stadium. Beide Stadien sollten als Montanvereisung im Hochgebirge begonnen haben, wobei die Eisscheide westlich des Gebirgskammes gelegen war. Danach wurde die Eisscheide nach Osten verschoben, so daß sie allmählich östlich der Fjällregion zu liegen kam. Die Ostverschiebung der Eisscheide war, nach LJUNGNeR's Ansicht, am stärksten während des jüngeren Stadiums. Außerdem meinte er, er könne komplizierte, durch Verschiebungen der Eiskulminationen hervorgerufene Drehungen hinsichtlich der Eisbewegungen feststellen. LJungnen's eigene Arbeit mit dieser Theorie wurde durch seinen vorzeitigen Tod (1954) abgebrochen. Andere Forscher, wie G. LundQvist und Holdar, verhalten sich skeptisch gegenüber der Möglichkeit, auf Grund der genannten Eisschrammenmethodik so weitgehende Schlußfolgerungen ziehen zu können, wie LJUNGNER es tat.

G. Wennberg (1949) brachte durch seine Umdeutung gewisser glazialgeologischer Verhältnisse einige Unruhe zustande. Er ging von theoretischen Erwägungen mit Rücksicht auf die Dynamik des Eises aus und prüfte seine Ideen in erster Linie an den Verhältnissen in Schonen. Er fand es dabei unmöglich, die herrschende Ansicht zu akzeptieren, daß ein baltischer Eisstrom eingedrungen sei, nachdem ein früheres sog. Nordosteis während einer Interstadialzeit abgeschmolzen sei. WENNBERG meinte, beide Eisströme wären gleichzeitig gewesen und hingen miteinander zusammen. Sie bildeten nach ihm einen $\mathrm{z} u-$ s a m m e hängenden E is kuchen, innerhalb dessen ursprünglich eine nordöstliche Richtung dominierte. Als diese allmählich aufhörte, sollte sich eine baltische Strömungsrichtung in einem Teil des Eiskuchens durch D if ferentia l bewe gung ausgebildet haben. Die Konsequenz dieser Hypothese war, daß Wennberg gezwungen wurde, viele Erscheinungen umzudeuten. Er mußte so in mehreren Fällen zur Theorie umfassender subglaziärer Sedimentation greifen, um das Vorhandensein oft mächtiger feinkörniger Sedimente unter und zwischen Moränenablagerungen zu erklären. Es war besonders diese Theorie, die heftig kritisiert wurde. ${ }^{5}$ ) Was die Verhältnisse in Schonen betrifft - WENNBERG hat versucht, seine Ideen ebenfalls auf andere Teile Skandinaviens anzuwenden - haben die Untersuchungen der letzten Jahre (Gunnar Johnsson 1956, 1958; Hans Möller, Mskr.; Kaj Nilsson, Mskr.) gezeigt, daß es keine wirkliche Interstadialzeit zwischen dem Nordosteis und dem baltischen Eis gegeben hat. Während Wennberg's Meinung in diesem Punkte bestätigt wird, zeigen allerdings die neuen Un-

4) Schwed. „häll“ wörtlich = Felsenfläche.

5) Siehe die Diskussion in „Geologiska Föreningens i Stockholm Förhandlingar“, Bd. 73, 1951, S. 316-318, 328-335. 
tersuchungen, daß das baltische Eis jedenfalls in großen Teilen Südwestschonens doch über eisfreiem Land vorgerückt sein muß. Der Zwang, eine umfassendere subglaziäre Sedimentation anzunehmen, fällt damit für Schonen fort.

Gösta Lundovist $(1948,1949)$ hat die Methode, Ablagerungsrichtung und Typ der Bodenart durch das Studium der Ein regelung der langgestreckten S teine in verschiedenen Bodenarten festzustellen, aufgenommen und weiter entwickelt. Diese Methode hat sich als sehr fruchtbar erwiesen und wurde von mehreren Forschern für verschiedene quartärgeologische $\mathrm{Z}_{\text {wecke }}$ angewandt.

U. a. hat Gunnar Hoppe (1952, 1957 usw.) dieselbe bei seinen bedeutungsvollen Studien über die Entstehung der kuppigen Moränenlandschaften angewandt. Er hat überzeugend gezeigt, daß subglaziäres Fließen von Grundmoränenmaterial - also nicht Obermoräne, wie früher oft angenommen wurde - eine große Rolle in diesem Zusammenhange gespielt hat. Unter dem Druck des Eises ist solches von Schmelzwässern durchtränktes Moränenmaterial in die subglaziären Hohlräume (Schmelzwasseraushöhlungen und dgl.) und Spalten des Eises hineingepreßt worden. Das Eis braucht übrigens nicht völlig „tot" gewesen zu sein. Der oft angewandte Ausdruck „Toteismoräne" würde also nicht immer völlig entsprechend sein. Große Teile des nördlichen Schwedens oberhalb der höchsten Küstenlinie bestehen aus solchen kuppigen Grundmoränenlandschaften, ebenso wie einige supraaquatisch gebildete Moränengebiete in Südschweden. Jahresmoränenähnliche Bildungen in diesen Gebieten haben nach Hoppe nichts mit wirklichen Jahresmoränen zu tun.

Ein anderes wichtiges Resultat der Forschungen Hoppe's und seiner Schüler bezieht sich auf die Eis abschmelzung im nördlichen Schweden. Nach der allgemeinen Auffassung soll das Eis in diesem Gebiet von der Eisscheide östlich der Fjällregion, wo es am mächtigsten war, zuletzt abgeschmolzen sein. Zwischen den Toteisresten an der Eisscheide und den Paßpunkten des Hochgebirges sollen große Eisseen aufgestaut worden sein. Was das nördlichste Norrland betrifft, hat jedoch C.-G. Holdar, ein Schüler Hoppe's, in einer neu erschienenen Arbeit (1957) klargelegt, daß ein Torne-Eissee, der früher nach SJöGREN im Schlußstadium der Eisabschmelzung im Torneträskgebiet vorgekommen sei, in Wirklichkeit nie existiert hat. Das Eis schmolz dort zuletzt im Hochgebirge ab, und Gelegenheit zur Bildung von Eisstauseen in großem Ausmaße gab es nicht.

Hoppe nimmt, wie auch LJUngner, eine große, umfassende Gebirgs verglets che rung während des Beginnes der letzten Vereisung an. Hierauf deutet das Vorkommen einer großen Anzahl niedrig gelegener Gletschernischen, die vor dem Maximum des letzten Eises gebildet wurden. Holdar spricht von einer Gebirgsvergletscherung sowohl zu Beginn als auch am Ende der Vereisung und von einem sog. Großeis (mit der Eisscheide östlich des Hochgebirges) bei dessen Maximum.

Man hat vermutet, daß außer in den Fjällgebieten ebenfalls in Südschweden weit ausgebreitete $\mathrm{E}$ is s t a u s e e n während der Abschmelzzeit vorgekommen sind (Erik NILSson). Bereits 1942 fragte sich Gösta LundQvist indessen, wo denn das Sediment der Eisseen vorhanden sei. Er betonte, daß Toteis im allgemeinen die tieferen Teile der vermuteten Eisseen eingenommen haben müsse, so daß höchstens nur randliche Partien zwischen dem Eis und den höhergelegenen Gebieten von offenem Wasser bedeckt waren. Sedimente konnten sich daher nur in lokalen, schmalen Landstrichen absetzen. Diese Auffassung scheint u. a. durch Untersuchungen im westlichen Südschweden von Gillberg (1956) bestätigt zu werden.

Von großer Bedeutung, u. a. für unsere Kenntnis des Klimas während spätglazialer Zeit, ist die Entdeckung von $\mathrm{E}$ is $\mathrm{ke}$ il e $\mathrm{n}$ i $\mathrm{n} \mathrm{S} \mathrm{ch} \mathrm{on} \mathrm{e} \mathrm{n.} \mathrm{Eiskeile} \mathrm{waren} \mathrm{früher} \mathrm{auf} \mathrm{nor-}$ dischem Gebiet nur in Dänemark (vor allem in Jütland westlich der ostjütländischen Endmoränen) bekannt. Die ersten Angaben über Eiskeile und kryoturbate Erscheinungen in Schonen wurden von Gunnar Johnsson (1956) gemacht. Während einige Phänomene, die 
von ihm als Frostfalten gedeutet wurden, bei anderen auf Widerspruch trafen, sind die Eiskeile allgemein auch von kontinentalen Experten (WOLDSTEDT, BüDEL) anerkannt worden. Die Eiskeile bestehen aus Frostspalten, die sich allmählich durch wiederholtes Gefrieren des Schmelzwassers erweiterten und dadurch die Form von Keilen mit nach unten sich allmählich verengender Spitze erhalten haben. Sie sind mit jüngeren Sedimenten gefüllt, die in den Keil eingesunken sind, als das Eis darin schmolz. Die einfachsten Typen der Eiskeile haben eine gleichbreite Strichform und haben sich allem Anschein nach durch Frost im Laufe eines einzigen Jahres bilden können. Für die Bildung der typischen, breiten Eiskeile ist eine bedeutend längere Zeit erforderlich. Eine Voraussetzung für die Ausbildung solcher Eiskeile ist das Vorhandensein von Dauerfrostboden. Die bisher beschriebenen Schonenschen Eiskeile wurden in der Umgebung von Landskrona (Saxtorp, Strandkliffe bei Glumslöv) und auf der Insel Ven gefunden. Die Eiskeile bei Saxtorp sind in glazifluvialem Schotter gebildet worden. Sie beginnen $1 / 2-1 \mathrm{~m}$ unter der Oberfläche und haben dort eine Breite, die bis zu $1 / 2 \mathrm{~m}$ werden kann. Die am besten entwickelten gehen bis zu einer Tiefe von $4 \mathrm{~m}$ hinunter. - Eiskeile verschiedenen Alters wurden angetroffen. Die jüngsten sind jünger als der baltische Eisstrom. Altere Eiskeile gibt es nach neuen, von JoHnsson ausgeführten Untersuchungen, teils zwischen NO-Moräne und baltischer Moräne, teils unter der NO-Moräne (Johnsson 1958). Die Funde der E is keile sind nicht nur in klimatologischer Hinsicht von Bedeutung, sondern a uch dadurch, daß sie eisfreies, nicht von Wasser bedecktes Gebiet für die Zeit der Eiskeilbildung angeben.

\section{Warvenchronologie}

Die Tonwarvenchronologie ist ja eine Forschung mit großer Tradition in Schweden. Gerard DE GEeR's Arbeit wird fortgesetzt von Frau Ebba Hult DE GEeR, die besonders mit einigen Arbeiten aus den Jahren 1954 und 1957 Übersichten und Gesichtspunkte mit Rücksicht auf die Geochronologie Skandinaviens gab. Im übrigen wird dieses Forschungsfeld dadurch charakterisiert, daß man mehr und mehr damit begonnen hat, K o n t r o ll messungen und Neumessungen der Warvenserien innerhalb gewisser kritischer Gebiete vorzunehmen. Bis jetzt sind nur einige wenige Berichtigungen das Resultat gewesen. So haben Borell \& Offerberg (1955) im Tal des Indalsälven herausgefunden, daß die Jahreszahl für die Abzapfung des zentraljäntländischen Eisstausees und die Bipartition des Inlandeises, also DE GEER's 0-Jahr, von diesem Forscher falsch konnektiert worden ist und in Wirklichkeit auf das Jahr 84 vor dem in der Praxis angewandten 0-Jahr fiel. Wenn man an DE GeER's Definition des 0-Jahres gleich dem Jahre der Bipartition festhalten will, so müßte also das 0-Jahr um die genannte Anzahl Jahre in der Zeitskala zurückverschoben werden. Die Verwendung des F o li e n k e r n b o h r e r s${ }^{6}$ ) hat es weiterhin möglich gemacht, daß man heutzutage leichter als früher Profile durch Bänderton bis hinunter zur untersten Warve erhalten kann. Warvenmessungen mit dem Folienkernbohrer wurden ausgeführt und sind an mehreren Stellen unseres Landes im Gange (Erik NiLsson, Björn JäRnEFons).

\section{Niveauveränderungen}

Die Frage der Niveauveränderungen ist ganz besonders umstritten. Hier stehen die Meinungen einander teilweise diametral gegenüber, und noch scheinen wir ein ganzes Stück von dem Ziel, diese Gegensätze zu überbrïcken, entfernt zu sein. Ich möchte mich daher im großen und ganzen nur damit begnïgen, Meinung gegen Meinung zu stellen.

Es ist natürlich wichtig, die höchste $\mathrm{K}$ üsten lin i e (oft als MG bezeichnet), an die das Meer und die großen baltischen Gewässer heranreichten, festzustellen. Hier scheint

6) Betr. dieses Bohrers siehe KJeLlman, Kallstenius \& Wager (1950). 
es klargelegt zu sein, daß viele Bestimmungen gar zu hoch sind. Dieses hängt offenbar mit den Schwierigkeiten zusammen, die höchste Küstenlinie von lokalen, höher gelegenen Strandlinien von Eisstauseen und dgl. zu trennen. Durch die Rücksichtnahme auf das Vorkommen von sog. Totcismoräne und glazifluvialen Schmelzwasserrinnen, die nicht unterhalb der höchsten Küstenlinie vorkommen, sind mehr restriktive Werte erhalten worden.

Ein überaus großes Beobachtungsmaterial zur Erforschung der Niveauveränderungen wurde in Westschweden, besonders im $\mathrm{T}$ al des $\mathrm{Flusses} \mathrm{Visk}$ a $\mathrm{n}$, von LenNarT von Post und seinen Schülern eingesammelt. Letzteres Gebiet wurde von L. von Posr ausgewählt als besonders geeignet $u$. a. für die Ermittlung der Rolle, die das eustatische Steigen des Meeres für den Verlauf der Niveauveränderungen gespielt hat. Es ist tragisch, daß von Post, der alle Fäden in seiner Hand hielt, nicht mehr die Zeit hatte, die Resultate seiner Viskan-Untersuchung vollständig zusammenzustellen. Einen kleinen Teil des Materials hat jedoch WENNER posthum veröffentlicht.

Die meisten Forscher, die sich dem Niveauveränderungsproblem gewidmet haben ich kann außer Lennart von Post (1947, 1948 und früher) Gunnar Gillberg (1956; Westschweden) und Erik NiLsson (1953, 1958; Sïdschweden) nennen - haben die Methode verwendet, ihre Strandlinienbeobachtungen entweder in Distanzdiagrammen oder in sog. Relationsdiagrammen zusammenzustellen. Eine Ausnahme bildet Sten FLORIN (1944-48), der in seinem Untersuchungsgebiet, Södermanland im östlichen Mittelschweden, so ungleichmäßige Niveauveränderungen in benachbarten Gegenden glaubt gefunden zu haben, daß er Strandliniendiagramme nicht verwenden konnte. Wesentlich verschiedene Schlußfolgerungen sind aus diesen Diagrammen herausgelesen worden. Ich kann jedoch hier nicht auf die evtl. Ursachen der Meinungsverschiedenheiten eingehen.

Auf der einen Seite haben von Post und Gillberg - wie im übrigen auch Florin aus seinem Material, nur von anderen Ausgangspunkten aus - se hr k o m plizie r te S trandverschiebung en während der spätquartären Zeit herausgelesen. Sie nehmen ein kompliziertes Zusammenspiel zwischen Veränderungen in der Höhenlage der Erdkruste (von Post's $\alpha$-Effekte) und den eustatischen Veränderungen des Meeresspiegels (von Post's $\omega$-Effekte) an. Die Erdkruste soll sich nicht gleichmäßig erhöht haben, wie früher eine Zeit lang allgemein angenommen wurde, sondern die Erhebung soll mehr oder weniger regelmäßig durch Retardation oder Landsenkung abgebrochen worden sein - von Post spricht in diesem Zusammenhang von elastischen Schocken, GiLlberg von Reversionen und Florin von $\mathrm{Und}$ ula tion en.

Im Gegensatz zu diesen Meinungen steht Erik NiLsson's Auffassung. Er meint, daß er aus seinem Material nurgleichmäßige und ruhige Niveauveränderungen ohne jegliche Schocke und ohne ausgeprägte Differentia 1 bewegungen zwischen den verschiedenen Schollen des Untergrundes ablesen kann. Ahnliche Ansichten vertreten Caldenius, Sandegren und Jan Lundovist.

Die Frage nach der Natur und der Zeitspanne des Ancylussees ist ein noch ungelöstes Problem. Wesentliche Neuigkeiten zur Erläuterung dieses Problemkomplexes sind von schwedischer Seite während des letzten Jahrzehnts kaum hinzugekommen.

Unsere Kenntnisse über die Áusdehnung des jetzt von Wasser bedeckten Gebietes, das früher einmal-bevor die postglaziale Transgression an der Westküste und die Ancylus- und Litorinatransgressionen im baltischen Gebiet ansetzten - $\mathrm{trock}$ en s $\mathrm{L}$ and war, sind durch neue Beobachtungen in der letzten Zeit erweitert worden. Carl CALdenius und Gunnel Linnman (1949) berichten von Funden fossiler Trockenkrusten, teils an der schwedischen Westküste (in einer Tiefe von $14 \mathrm{~m}$ bei Halmstad), teils im Ostseegebiet (in einer Tiefe von $6 \mathrm{~m}$ bei Karlshamn). 
Kombinierte dänisch-schwedische Untersuchungen mit dem Kullenberglot haben jedoch das Vorkommen festen Landes während spätquartärer Zeit in größerer Tiefe der südlichen Ostsee nicht bestätigen können (Kullenberg 1954). Die bekannten Kåseberga-Kiefernstümpfe, die in einer Tiefe von ca. $35 \mathrm{~m}$ südlich Schonens angetroffen wurden, und einige andere ähnliche Funde vom Grunde der Ostsee gaben Anlaß zu dieser Annahme. Nach KuLLF,NBERG scheinen die bisher gemachten Erfahrungen eher zu zeigen, daß das Gebiet unterhalb $30 \mathrm{~m}$ Tiefe in der südlichen Ostsee immer von Wasser bedeckt war. Keine Trockenkruste, keine Kiefernstümpfe oder lakustrinen Sedimente sind angetroffen worden. Näher der Schonenschen Küste besteht jedoch der Meeresboden aus Sand, der auf harter Moräne ruht.

Die Stratigraphie der marinen Ton e wurde von Fritz Brotzen (1951) angegriffen. Er hat festgestellt, daß die Foraminiferenfauna der marinen Tone im westlichen Südschweden von Schicht zu Schicht wechselt. Wirkliche Leitformen wurden von ihm aufgestellt, die deutlich spätglaziale und postglaziale Tone trennen.

\section{Vegetations - und Klimageschichte}

In einem großen Teil der Schriften, die die Niveauveränderungen behandeln, wurde die Altersbestimmung wenigstens gewisser kritischer Punkte durch Verwendung der Pollen a n l yse ausgeführt. Für rein vegetationsgeschichtliche Untersuchungen sowie für das Lösen der torfgeologischen und klimageschichtlichen Probleme ist die genannte Methode mehrfach angewandt worden. Nunmehr hat man in vielen Fällen - hauptsächlich nach dänischem Vorbild - damit begonnen, eine mehr fortgeschrittene Form der Pollenanalyse zu verwenden. Während man sich früher hauptsächlich mit der Zählung des Baumpollens begnügte, nimmt man nunmehr in immer größerem Ausmaße Rücksicht auch auf den Blütenstaub der Sträucher und Kräuter, um ein so vollständiges Bild wie möglich von der Vegetation und deren Veränderungen zu bekommen. Die Möglichkeit, Pollendiagramme zu konnektieren und datieren, nimmt auch bis zu einem gewissen Grade in gleichem Maße wie die Anzahl der Pollenarten zu.

Rein vegetationsgeschichtlichen $Z_{\text {weck }}$ haben einige größere Arbeiten von Magnus Fries (1951, 1958). In der ersten dieser Arbeiten meint FrIEs, er könne Allerödschichten im mittleren Bohuslän an der schwedischen Westküste erkennen. Das Optimum der postglazialen Wärmezeit, die Hochwärmezeit, verlegt er auf den späteren Teil der mesolithischen Zeit, einen Abschnitt, der waldgeschichtlich durch die Linde charakterisiert wird. Bereits zu Beginn des Neolithikums meint er Anzeichen schlechteren Klimas erkennen zu können. Die Zeit der postglazialen Klimaverschlechterung rechnet er vom genannten Zeitpunkt an. In der Arbeit von 1958 versucht Fries Spuren der Einwirkung der Kultur auf die Vegetation im Pollendiagramm zu finden (vgl. auch Maj-Britt FloRIN 1957).

Bei einer Untersuchung eines Moores auf der Tönnersjöheide im südlichen Halland ist es Olausson (1957) gelungen, zum ersten Mal das Vorkommen von fossilem Ilex in Schweden nachzuweisen. In Schichten der Hochwärmezeit traf er auf drei Pollenkörner dieser Pflanze.

In Schonen und Blekinge sind mehrere größere torfgeologisch-vegetationsgeschichtliche Untersuchungen im Gange. Teilweise liegen bereits die Resultate in Manuskriptform vor (Björn Berglund, Ernest MaGnusson). In dem großen Moorkomplex nordwestlich des Sees Ringsjön, vor allem im Agerödsmosse ${ }^{7}$ ), wurden von C. A. Althin reiche mesolithische Kulturschichten ausgegraben. Die Bearbeitung der Torfproben dieser Ausgrabung und von besonders eingesammeltem Material zur Darstellung der Entwicklungsgeschichte des Moores wird gegenwärtig am Quartärgeologischen Institut der Universität Lund betrieben.

7) Schwed. „mosse“ $=$ Moor. 


\section{Altersbestimmungen mit der Radiokarbonmethode}

Altersbestimmungen mit der Radiokarbonmethode scheinen in einer glücklichen Weise die Pollenanalyse als Datierungsmethode zu ergänzen. Wie schon vorher gesagt wurde, sind eine Reihe von $\mathrm{C}^{14}$-Bestimmungen an Funden, die offenbar interglazial sind, ausgeführt worden. Für spätquartäre Ablagerungen sind weiterhin bereits eine ganz erhebliche Anzahl solcher Datierungen veröffentlicht worden (O'STLUND, GEJvall \& LuNDQvist 1956; G. LundQvist 1957; Jan LundQvist 1957, 1958; ÓstLund 1957; M. Fries 1958). Einige der Ergebnisse möchte ich hier anführen. Alle Werte im folgenden werden in Jahren v. Chr. angegeben.

Bei Roberts dal im südlichen Schonen liegt offenbar eine Allerödablagerung vor: Alter $9025 \pm 140$ v. Chr. (G. Lundqvist).

Die $s$ ubmarinen Ki ef e r nstü m p fe, die aus einer Tiefe von ca. $35 \mathrm{~m}$ außerhalb von $\mathrm{K}$ å s e b e rg a aufgedreggt wurden, haben ungefähr das richtige Alter, das sie haben sollten, wenn sie auf der Stelle gewachsen wären, wo sie angetroffen wurden. Eine $\mathrm{C}^{14}$-Bestimmung ergab den Wert $7375 \pm 120 \mathrm{v}$. Chr. Etwa gleichen Alters ist ein Kiefernstumpf, der außerhalb $\mathrm{K}$ a r $1 \mathrm{sk}$ ron a s aus einer Tiefe von $38 \mathrm{~m}$ aufgefischt wurde: $7145 \pm 120$ v. Chr. (G. Lundevist).

Ein $S \mathrm{trandwall}$ a us der Litorinazeit bei Ramsås, nördlich von Kalmar, entstand nach dem Jahre $5075 \pm 110$ v. Chr., wie aus einer C $C^{14}$-Bestimmung der unterlagernden Torfschicht hervorgeht (G. LundQvist). Diese Datierung stimmt sehr gut mit einer mit anderen Methoden ausgeführten Altersbestimmung der ersten Litorinatransgression weiter im Süden, in Schonen, überein (ca. 5000 v. Chr.; T. NILsson 1935).

Die prägnante Ulmus-Abnahme im mittleren Postglazial, am Ende der eigentlichen Hochwärmezeit nach Fries, wurde an einigen Vorkommen in Schweden mit der Radiokarbonmethode datiert: 1) bei M o g e to r p in Södermanland (Durchschnittszahl dreier einander sehr nahegelegener Werte: ca. 3400 v. Chr.; Sten Florin, 1958); 2) an zwei Stellen in der Umgegend von Varnhem in Västergötland (3330 \pm 110 v. Chr. bzw. $3630 \pm 110$ v. Chr.; Fries 1958). Alle drei Bestimmungen stimmen auffallend gut überein. Sie ergeben die Durchschnittszahl ca. 3430 v. Chr. (vgl. die Jahreszahl 3500 v. Chr. bei T. NiLsson 1935, eine Zahl, die durch die Berechnung der durchschnittlichen Zuwachsgeschwindigkeit der organogenen Schichtenfolgen in Schonen erhalten wurde).

Weiterhin ist zu bemerken, daß eine deutliche Verspätung der Einw a nderung der Fichte von Nord- nach Südschweden nachgewiesen werden kann. Während der markante Anstieg der Fichte etwa 1000 v. Chr. in Nordschweden stattfindet, geschieht dasselbe im westlichen Mittelschweden (Värmland) erst einige hundert Jahre später, ca. 200-500 v. Chr. (Jan Lundevist).

Zum Schluß möchte ich einige neue $C^{14}-\mathrm{D}$ a tierungen a us Südschwed e n anführen. Diese wurden von Göte Óstlund am C14-Laboratorium in Stockholm (Proben mit der Bezeichnung St; vgl. Óstuund 1959) und von Ingrid Olsson am Physikalischen Institut der Universität Uppsala (Proben mit der Bezeichnung U) ausgeführt.

Bei T o p pela dugå r , ca. $15 \mathrm{~km}$ im SO von Lund, gibt es ein klassisches Vorkommen von Allerödschichten, die bereits von HoLst (1906) behandelt und später von mir pollenanalytisch untersucht wurden ( $T$. NiLsson 1935). Im Frühjahr dieses Jahres wurden Proben zur C $\mathrm{C}^{14}$-Datierung dieses Allerödvorkommens genommen. Die Allerödschichten, welche von etwa $2 \mathrm{~m}$ jüngerem Dryaston bedeckt sind, bestehen: 1) zuoberst aus einer dünnen Schicht Kalkgyttja; 2) darunter aus Tongyttja, die in einen oberen und einen unteren Horizont durch ein $Z$ wischenlager von gyttjahaltigem Ton aufgeteilt ist; 3) zuunterst aus einer dünnen Schicht von torfartigem sog. Allerödmull. Die Kalk- 
gyttja hinterließ nach der Entfernung des Kalkes mit Säure viel zu wenig organische Úberreste für eine $\mathrm{C}^{14}$-Datierung. Der obere Tongyt tjahorizont ergab den Wert $9940 \pm 180$ v. Chr. (St-345), d e r A 1le rö d m u 11: $10040 \pm 200$ v. Chr. (St-341). Diese Werte lassen sich ganz gut mit den dänischen $\mathrm{C}^{14}$-Datierungen der Allerödschichten in Ruds Vedby vergleichen. Dort wurden die folgenden Jahreszahlen erhalten: $8880 \pm$ 200 v. Chr. für das Ende des Alleröd; $9020 \pm 220$ v. Chr. bis $9930 \pm 340$ v. Chr. für das mittlere Alleröd und den letzten Teil des älteren Alleröd (Iversen 1953). D e r Allerödmull in Toppeladugård dürfte etwa den Beginn der Allerödzeit angeben.

Aus dem südöstlichen B leking e sind zwei Proben, die von Björn Berglund eingesammelt wurden, C14-bestimmt worden.

Im nordwestlichen Teil des kleinen Sees F ärsks jö n, der auf der Torhamnhalbinsel (14 $\mathrm{m}$ ü. M.) gelegen ist, fand Berglund auf dem Grunde des Sees eine ausgebreitete Stubbenschicht, die von ungefähr $1 \mathrm{~m}$ Wasser bedeckt war. Der Abfluß des Sees ist im Süden gelegen. Die vorliegenden Umstände deuten auf e rhöhten Wasserstand im See aufgrund einer Klimaverschlechterung mit vermehrtem Niederschlag, wodurch der Wald an den Rändern ertränkt wurde. Eine C14-Datierung eines der Stümpfe (eines Kiefernstubbens) ergab die Jahreszahl $1950 \pm 80$ v. Chr. (St-313). Das Ansteigen des Wasserstandes kann mit einer Zunahme des Niederschlages in Verbindung gebracht werden, die allgemein u. a. in Schonen festgestellt wurde und die dort die Ursache einer der ausgeprägtesten Rekurrenzflächen war (T. NILSson 1935). Diese wurde etwa am Ende der Ganggräberzeit ausgebildet und kann mit GranLund's RY V verglichen werden.

Die andere Probe ist ein Schwemmtorf, reich an kleineren Hölzern, von einer Meeresbucht, Sörevik, südöstlich von Karlskrona. Der $\mathrm{Schwemm}$ torf befand sich in einer Tiefe von 4-7 m unter dem Wasserspiegel zwischen unterlagerndem Feinsand und deckenden Schichten von gyttjahaltigem Ton und Gyttja. Gemäß der Pollenanalyse gehört dieser Schwemmtorf zum untersten Teil der Zone VIII im Schonenschen Zonenschema, in der Nähe des Überganges zur Zone IX. Die Zonengrenze VIII/IX dürfte in der baltischen Entwicklung etwa dem Übergang der Yoldiazeit zur Ancy$1 \mathrm{usz}$ e it entsprechen, einem Zeitpunkt, den man etwa 7500 v. Chr. oder etwas später anzusetzen pflegt. Die C $\mathrm{C}^{14}$-Bestimmung des Schwemmtorfes von Sörevik ergab die Jahreszahl $7040 \pm 140$ v. Chr. (St-333). Gemäß dieser Altersbestimmung sollte der Schwemmtorf bei einer Tiefe von 4-7 $\mathrm{m}$ nur wenige hundert Jahre jünger sein als die soeben genannten Kiefernstümpfe, die in bedeutend tieferem Wasser angetroffen wurden. Das Ansteigen des Wasserstandes im südlichen Ostseegebiet scheint während dieser Zeit schnell vor sich gegangen zu sein.

Schließlich wurden ebenfalls einige Proben von a r chä ologis chem Material (von Holger Arbmann eingeliefert) $\mathrm{C}^{14}$-datiert. Da diese Bestimmungen auch von geologischem Interesse sind, führe ich sie hier an.

1) Vät te ryd (Mittelschonen): Holzkohle aus einer Kulturschicht der Dolmenzeit. $C^{14}$-Alter zweier Proben: $2595 \pm 140$ v. Chr. (U-46) bzw. $2730 \pm 170$ v. Chr. (U-47).

2) E 1 in el u n d (bei Limhamn): Holzkohle aus einer Kulturschicht der späten Erteböllezeit. C14-Alter: $3360 \pm 210$ v. Chr. (U-48).

Letztgenannte Datierung ist von unmittelbar geologischem Interesse, da die fragliche Kulturschicht - aus der späteren Erteböllezeit - auf der Innenseite des Litorinastrandwalles, dem sog. Järawall, lag und von diesem noch überlagert war. Der Järawall war also um die Zeit $3360 \pm 210$ v. Chr. bei Malmö noch nicht fertig ausgebildet. Dieses besagt jedoch nichts prinzipiell Neues. Durch Otto RYDBECK's Untersuchungen wissen wir, daß der Litorinagrenzwall im südlichen Schonen in seinen obersten Teilen aus der Ganggräberzeit stammt. 


\section{Zitierte Literatur ${ }^{8}$ )}

Abkürzungen: GFF $=$ Geologiska Föreningens i Stockholm Förhandlingar

SGU $=$ Sveriges Geologiska Undersökning, Stockholm

BORELl, R. \& OfFERBERG, J., 1955: Geokronologiska undersökningar inom Indalsälvens dalgång mellan Bergeforsen och Ragunda. (Geochronological investigations in the Indal River valley between Bergeforsen and Ragunda, N. Sweden.) - SGU, Ser. Ca. 31.

BrotZen, F., 1951: Bidrag till de svenska marina kvartäravlagringarnas stratigrafi. (Contributions to the stratigraphy of the marine Quaternary sediments of Sweden.) - GFF 73.

Caldenius, C. \& Linnman, GunNel, 1949: En senkvartär regressions- och transgressionslagerföljd vid Halmstad. - SGU, Ser. C. 502.

De GeER, EbBa Hult, 1954: Skandinaviens geokronologi. (With an abstract in English.) - GFF 76. - - 1957: Old and new datings of Swedish ice lakes and the thermals of Bölling and Alleröd. - GFF 79.

Florin, Maj-Britt, 1957: Pollen-analytical evidence of prehistoric agriculture at Mogetorp Neolithic settlement, Sweden. - In: S. Florin, Vråkulturen. Stockholm, K. Vitterh. Hist. Antikv. Akad., Monogr., 1958.

FLORIN, S., 1944-48: Havsstrandens förskjutningar [Kustförskjutningen] och begyggelseutvecklingen i östra Mellansverige under senkvartär tid. (Die Strandverschiebungen im östlichen Mittelschweden in spätquartärer Zeit.) 1-2.-GFF 66, 70. - - 1958: Vråkulturen, Stenåldersboplatserna vid Mogetorp, Östra Vrå och Brokvarn. - K. Vitterh. och Hist. Antikv. Akad., Monogr., 300 S., 53 Taf., Stockholm.

FrIEs, M., 1951: Pollenanalytiska vittnesbörd om senkvartär vegetationsutveckling, särskilt skogshistoria, i nordvästra Götaland. (Pollenanalytische Zeugnisse der spätquartären Vegetationsentwicklung, hauptsächlich der Waldgeschichte, im nordwestlichen Götaland [Südschweden].) - Acta Phytogeogr. Suec. 29. Uppsala. - - 1958: Vegetationsutveckling och odlingshistoria i Varnhemstrakten. En pollenanalytisk undersökning i Västergötland. (Vegetationsentwicklung und Siedlungsgeschichte im Gebiet von Varnhem. Eine pollenanalytische Untersuchung aus Västergötland [Südschweden].) - Ibid. 39.

Gillberg, G., 1956: Den glaciala utvecklingen inom Sydsvenska höglandets västra randzon. 3. Issjöar och isavsmältning. (The development of the deglaciation in the western marginal zone of the South-Swedish Highlands.) - GFF 78.

Holdar, C.-G., 1957: Deglaciationsförloppet i Torneträskområdet efter senaste nedisningsperioden. (With an English summary.) - GFF 79.

Holst, N. O., 1906: De senglaciala lagren vid Toppeladugård. - SGU, Ser. C. 210.

HopPE, G., 1952: Hummocky moraine regions, with special reference to the interior of Norrbotten. - Geogr. Ann. 34, Stockholm. - - 1957: Problems of glacial morphology and the Ice Age. - Ibid. 39.

Hoppe, G. \& LiLJEQuist, G. H., 1956: Det sista nedisningsförloppet i Nordeuropa och dess meteorologiska bakgrund. (The course of the last glaciation in northern Europe and its meteorological background.) - Ymer 76, Stockholm.

Iversen, J., 1953: Radiocarbon dating of the Alleröd Period. - Science 118, Washington.

JÄRNEFORS, B., 1956: Isrecessionen inom Uppsalaområdet. (Ice recession in the Uppsala region.) GFF 78 .

Johnsson, G., 1956: Glacialmorfologiska studier i södra Sverige. (Glacial morphology in southern Sweden.) - Lund, Univ. Geogr. Inst., Avh. 31. - - 1958: Submoraine icewedges in Western Scania. - GFF 80.

KJellman, W., Kallstenius, T. \& Wager, O., 1950: Soil sampler with metal foils. Device for taking undisturbed samples of a very great length. - Stockholm, Roy. Swed. Geotechn. Inst., Proc. 1.

Kullenberg, B., 1954: On the presence of sea water in the Baltic Ice-Lake. - Tellus 6, Stockholm. LINDROTH, C., 1948: Interglacial insect remains from Sweden. - SGU, Ser. C. 492.

LJUNGner, E., 1949: East-west balance of the Quaternary ice caps in Patagonia and Scandinavia. - Uppsala, Geol. Inst., Bull. 33.

LUNDQvist, G., 1942: Var äro issjöarnas sediment? - GFF 64. - - 1948: Blockens orientering i olika jordarter. (With an abstract in English.) - SGU, Ser. C. 497. - - 1949: The orientation of the block material in certain species of flow earth. - Geogr. Ann. 31, Stockholm. - 1951: Beskrivning till jordartskarta över Kopparbergs län. - SGU, Ser. Ca. 21. - 1955: Stocken i OOje. Ett säkert interglacialt fynd. (With an abstract in English.) GFF 77. - - 1957: C 14-analyser i svensk kvartärgeologi 1955-57. (With an English summary.) - SGU, Ser. C. 557. - - 1958: Beskrivning till jordartskarta över Sverige. SGU, Ser. Ba. 17.

8) Die angeführten Schriften des letzten Jahrzehnts sind nur eine Auswahl der während dieser Zeit erschienenen. 
Lundqvist, G. \& Granlund, E., 1957: De kvartära bildningarna. - In: N. H. Magnusson, G. LundQvist \& E. Granlund, Sveriges geologi. 3. Aufl., Stockholm.

LUNDQVIST, J., 1955: Interglacialfyndet vid Boliden. (With an abstract in English.) - GFF 77. - 1957: C 14-dateringar av rekurrensytor i Värmland. (C 14-determinations of recurrence surfaces in Vermland, western Sweden.) - SGU, Ser. C. 554. - - 1958: Beskrivning till jordartskarta över Värmlands län. (Quaternary deposits of the County of Värmland.) - SGU, Ser. Ca. 38.

MaGnusson, N. H., 1958: Sveriges geologiska undersökning inför sitt 100-års-jubileum. - Svensk naturvetenskap, 1957-1958, Stockholm.

MARKGREN, M., 1956: Glaciation och landskap i norra Fennoskandia. (Glacierization and landscape in N Fennoskandia.) - Svensk geogr. Årsb. 32, Lund.

Matrsson, Å., 1954: Isräfflornas användbarhet för tolkningen av nedisningsförloppet. (Die Verwendbarkeit der Eisschrammen, um den Vereisungsverlauf zu deuten.) - Ibid. 30.

NILsson, E., 1953: Om södra Sveriges senkvartära historia. (With an English summary.) - GFF 75. - - 1958: Issjöstudier i södra Sverige. (With an English summary.) - GFF 80.

NiLsson, T., 1935: Die pollenanalytische Zonengliederung der spät- und postglazialen Bildungen Schonens. - GFF 57.

Olausson, E., 1957: Das Moor Roshultsmyren. Eine geologische, botanische und hydrologische Studie in einem südwestschwedischen Moor mit exzentrisch gewölbten Mooselementen. Lund, Univ. Årsskr., N. F. (2) 53.

ỚstLund, H. G., 1957: Stockholm natural radiocarbon measurements I.-Science 126, Washington. - - 1959: Stockholm natural radiocarbon measurements II. - Amer. J. Sci. 257, New Haven (im Druck).

Ớstlund, G., Gejvall, N.-G. \& Lundqvist, G., 1956: Åldersbestämning med hjälp av kol-14. Ymer 76, Stockholm.

voN Post, L., 1947: Hallands marina fornstränder. $\alpha$ och $\omega$ vid Nordens senkvartära nivåförändringar. - GFF 69. - - 1948: Ovre Klarälvsdalens fornfjord. (With an abstract in English.) GFF 70. - 1955-56: The ancient sea fiord of the Viskan valley, 1-2. (Ed. by C.-G. WENNER.) - GFF 77-78.

RYDBECK, O., 1928: Stenåldershavets nivåförändringar och Nordens äldsta bebyggelse. (The changes of level of the Stone Age Sea and the earliest settling of man in Scandinavia.) Lund, K. Humanist. Vetenskapssamf., Årsber. 1927-28.

SANDEGREN, R., 1947: Svenska nivåförändringsproblem. - Köbenhavn, Dansk Geol. Foren., Meddel. 11.

Wennberg, G., 1949: Differentialrörelser i inlandsisen. Sista istiden i Danmark, Skåne och Ơstersjön. (Mit Zusammenfassung in deutscher Sprache.) - Lund, Univ. Geol.-Min. Inst., Meddel. 114.

Manuskr. eingeg. 3. 12. 1958.

Anschrift des Verf.: Dr. Tage Nilsson, Quartärgeologisches Institut der Universität Lund, Sölvegatan 13, Lund, Schweden. 